\title{
Spatially Embedded Dynamics and Complexity
}

\author{
Christopher L. Buckley ${ }^{\mathrm{a}}$, Seth Bullock ${ }^{\mathrm{b}}$, Lionel Barnett ${ }^{\mathrm{a}}$ \\ ${ }^{a}$ Centre for Computational Neuroscience and Robotics, University of Sussex, UK \\ ${ }^{b}$ School of Electronics and Computer Science, University of Southampton, UK
}

\begin{abstract}
To gain a deeper understanding of the impact of spatial embedding on the dynamics of complex systems we employ a measure of interaction complexity developed within neuroscience using the tools of statistical information theory. We apply this measure to a set of simple network models embedded within Euclidean spaces of varying dimensionality in order to characterise the way in which the constraints imposed by low-dimensional spatial embedding contribute to the dynamics (rather than the structure) of complex systems. We demonstrate that strong spatial constraints encourage high intrinsic complexity, and discuss the implications for complex systems in general.
\end{abstract}

Key words:

complexity, space, networks, information theory

\section{Introduction}

From its outset, complexity science has concentrated on how simple properties can give rise to complex organisation and behaviour. The interplay between, inter alia, non-linear, local interactions, physical constraints, noise, and processes of copying or competitive exclusion have been shown to give rise to self-organisation, auto-catalysis, path dependence, and emergent behaviour in many different ways [20, 19]. Most real-world complex systems are spatially extended systems. For example, nervous systems, ecologies, economies, cities, etc., all exhibit multiple scales of spatial organisation. The impact that this spatial embedding has on the behaviour of these systems is not well-understood. In particular, the role of spatial constraints in influencing the ability of these systems to exhibit organised behaviour is an open question. 
It is clear that projecting a system of interacting elements into a low dimensional space such that interactions tend to occur only between elements that are near to one another must restrict the possible ways in which the system elements can be "connected". This restriction might be viewed as a frustrating constraint that prevents, or at least mitigates against, useful kinds of organisation that rely upon "long-range" connections. However, embedding a system's interactions within a space also imposes potentially useful local correlations and symmetries on its organisation "for free". In fact, studies show that spatial embedding of this kind can predispose systems to exhibit behaviour that would otherwise be unstable $[8,14]$. Might the constraints imposed by low-dimensional spatial embeddings actually be critical to enabling complex systems to arise and persist? This notion casts spatial constraints as potentially "enabling" rather than frustrating, in that they might naturally steer or bias a system's organisation in a potentially useful fashion [11].

Here we are interested in the relationship between spatial constraints, network topology, and interaction complexity, an information-theoretic measure developed in order to characterise the way in which both vertebrate and invertebrate nervous organisation might influence nervous function [34]. Before we introduce this measure, we first briefly describe some examples of the way in which space can influence network dynamics. By applying an approximation of the interaction complexity measure to a simple spatially embedded network and varying the dimensionality of the space, we characterise and explain the relationship between the structural properties conferred by spatial embedding and any attendant influence on interaction complexity. We conclude with a discussion of the implications for complex systems in general.

\section{Networks in Space}

The recent explosion of interest in the "new science of networks" [22, 7, 21] has focused attention on the application of graph-theoretic approaches to the characterisation of natural and engineered systems. While the influence of space was at least implicit in certain of the first graph structures discussed and employed in this literature, its contribution has only started to be systematically explored relatively recently $[23,4]$.

For instance, Stanley Milgram's now infamous demonstration of the "six degrees of separation" that apparently link members of society to each other 
through mutual acquaintance relies upon space [37]. The instruction to each of Milgram's experimental subject was to deliver a package to a person identified only by name and place of residence. Subjects were thus clearly required to combine their social and geographical knowledge to meet this challenge. The role of spatial knowledge and the spatial structure of social networks is not often recognised in discussion of the surprisingly short routes that the successfully delivered packages took.

Here we wish to explicitly explore the relationship between spatial embedding and the properties that it confers on both the network topology of complex systems and their consequent behaviour. Several modelling studies suggest that this relationship may be significant. We describe two below.

Boerlijst and Hogeweg [8] demonstrate the power of spatial embedding in their model of molecular self-organisation in "hypercycles" [15]. A hypercycle comprises a set of molecular species, where each species supports the persistence of some of the others and, in total, they achieve the persistence of the entire set. While such organisations appear to offer a route by which persistent co-operative collaboration might arise spontaneously, Such organisations can be parasitised by free-riding molecular species that benefit from interacting with some member(s) of the hypercycle, but do not support the hypercycle's persistence in return. In Boerlijst and Hogeweg's model parasitisation of this kind destroyed hypercycles when the population was well-mixed (i.e., non-spatial). When the same system of molecular species was embedded within a lattice such that individual molecules could only interact with their close spatial neighbours, the hypercycles that arose were spatially organised as rotating spirals and were also able to resist parasites.

Di Paolo [14] shows the importance of spatial embedding for complex organisation in a somewhat different context. He shows that an altruistic behaviour is unstable in a well-mixed non-spatial model: exploitation quickly undermines any tendency towards co-operation. However, the same altruistic behaviour is prevalent in the same model when individuals are distributed across a two-dimensional continuous plane. When their interactions are spatially constrained, individuals spontaneously organise into clusters of altruistic individuals, each surrounded by an annulus of non-altruists.

In both of these studies, systems were able to achieve a sophisticated mode of functional organisation only when they were embedded within a low-dimensional space. No such organisation could persist when the system's components were entirely well mixed and its interactions were, as a consequence, unconstrained. What of systems that lie between these two 
extremes? The notion of exploring systems that lie between order (e.g., a lattice) and disorder (e.g., a random graph) is familiar within complexity science since complexity measures typically seek to capture the nature of systems that are neither completely random nor completely regular. A familiar rhetorical device is taken from the statistical mechanics of gases and crystals. While the low-level organisation of a gas can be idealised as random and that of a crystal can be idealised as regular, the aggregate behaviour of each is readily derivable. For intermediate systems at the phase transition between solid and fluid, however, this relationship is less clear. Complexity, it is claimed, exists in this middle ground between order and disorder [19].

Here, we explore the behaviour of systems that lie between the two extremes reported by Di Paolo and Boerlijst and Hogeweg by relaxing the constraints imposed by spatial embedding through increasing the dimensionality of the metric space within which the network nodes are located. As the dimensionality increases (while the density of connections is held constant) the spatially imposed correlations amongst the system's interactions diminish, until, in the limit of an infinite dimensional space, a random, uncorrelated graph is achieved, equivalent to a well-mixed system.

Since we are interested in the complexity of the interactions between the elements described by such a network, rather than the structure of the network itself, we employ of measure of interaction complexity developed within neuroscience and described in the following section.

\section{Interaction Complexity}

Central to cognitive processing within the nervous system is the ability of the brain to integrate distributed information in order to produce coherent cognitive behaviour. For example, information from audio, visual and olfactory input must be successfully integrated and used to inform subsequent motor output [34]. In contrast, a great deal of experimental work demonstrates that separate neural regions are specialised and hence quasiindependent. For instance, in the mammalian brain different neural areas are functionally specialised for detection of visual attributes such as shape, motion and colour. ${ }^{1}$ Neural systems must balance this functional segregation

\footnotetext{
${ }^{1}$ It is interesting to note that neuroscientists have been convinced that spatial organisation of this kind is implicated in the complexity of neural behaviour since the first staining technologies began to reveal the structure of animal brains $[40,27]$ and that spa-
} 
at the level of neural modules with the requirement for functional integration at the level of the organism. Tononi, Sporns and Edelman [34] proposed an interaction complexity measure that captures this tension within a single metric.

In this paper we will refer to this measure as TSE complexity. We describe it as a measure of interaction complexity since it is principally concerned with capturing the nature of the interactions amongst parts of a system rather than the static structure of the system, per se. Despite being conceived within a specific neuroscience context, TSE complexity has received widespread attention across the behavioural and brain sciences, from fields as diverse as autonomous robotics [26], neural imaging [16, 30], local dynamics of the mammalian brain [28] and the exploration of theories of sleep, consciousness and schizophrenia [32].

TSE complexity is derived (see [34, 35, 36, 29]) by considering an isolated set of $n$ "neural components" (nodes for brevity) and a stationary multivariate stochastic process $\mathbf{X}(t) \equiv\left\{X_{i}(t) \mid i=1, \ldots, n\right\}$ running on the system, where $X_{i}(t)$ is to represent the activation state at time $t$ of the $i^{\text {th }}$ node. Firstly the integration associated with the system is introduced:

$$
\mathcal{I} \equiv \sum_{i=1}^{n} H_{i}-H
$$

where $H$ denotes the entropy $H(\mathbf{X}(t))$ of the full process $\mathbf{X}(t)$ and $H_{i}$ the entropy $H\left(X_{i}(t)\right)$ of the individual activation $X_{i}(t)$. Note that by stationarity these quantities and hence $\mathcal{I}$ itself do not depend on time $t$. $\mathcal{I}$ may be interpreted as a measure of the deviation from independence of the individual components of the system. TSE complexity is then defined to be:

$$
\begin{aligned}
\mathcal{C} & \equiv \sum_{k=1}^{n-1}\left(\frac{k}{n} \mathcal{I}-\langle\mathcal{I}\rangle_{k}\right) \\
& =\sum_{k=1}^{n-1}\left(\langle H\rangle_{k}-\frac{k}{n} H\right)
\end{aligned}
$$

where $\langle\cdot\rangle_{k}$ denotes an average over all subsystems of size $k$. In the special case

tial constraints have recently been invoked to account for the circuit complexity of cortical structures [12]. 
where the $\mathbf{X}(t)$ are multivariate Gaussian, the entropy $H$ may be expressed simply in terms of the $n \times n$ covariance matrix $\Omega \equiv \overline{\mathbf{X}(t)^{\top} \mathbf{X}(t)}$, where the over-bar represents an average over the statistical ensemble [13]. Again, by stationarity $\Omega$ does not depend on time $t$. We then have $H=\frac{1}{2} \ln \left([2 \pi e]^{n}|\Omega|\right)$ so that:

$$
\mathcal{C}=\frac{1}{2} \sum_{k=1}^{n-1}\left(\langle\ln |\Omega|\rangle_{k}-\frac{k}{n} \ln |\Omega|\right)
$$

Like other notions of complexity, this measure is low when either all elements are independent and hence completely segregated, or the system is completely integrated. Complexity is maximal in a system that is globally integrated at the level of large subsystems, but simultaneously exhibits a high degree of segregation between smaller subsystems.

Tononi et al. [34] consider an $n \times n$ connectivity matrix, $C$, where $C_{i j}$ is to be interpreted as the weight on the connection from (efferent) node $i$ to (afferent) node $j$, and a linear regressive neural process $\mathbf{X}(t)$ driven by uncorrelated Gaussian noise. However, there is an error in their calculation of the covariance matrix. In [2] this error is corrected via introduction of the continuous time multivariate Ornstein-Uhlenbeck process [38]:

$$
d \mathbf{X}(t)=-\mathbf{X}(t) \cdot(I-C) d t+d \mathbf{W}(t)
$$

where $I$ is the identity matrix and $\mathbf{W}(t)$ a multivariate Wiener process with identity covariance matrix ${ }^{2}$. Eq. (5) may be viewed as a linearised, noisy Continuous Time Recurrent Neural Network (CTRNN) with $I$ corresponding to a leak current term $[5,6,17]$. $\mathbf{X}(t)$ will then be multivariate Gaussian so that (4) applies. The condition for stationarity of (5) is shown to be:

$$
\mathfrak{R e}(\lambda)<1 \text { for every eigenvalue } \lambda \text { of } C
$$

and the covariance matrix $\Omega$ is shown to satisfy:

$$
2 \Omega=I+C^{\top} \Omega+\Omega C
$$

Several subsequent statistical measures derived from information theory have attempted to quantify properties analogous to complexity. These in-

\footnotetext{
${ }^{2}$ Note that the noise input to different nodes is uncorrelated. If we allow noise levels to differ per node, then we may recover an equivalent equation to (5) by a simple linear transformation of the connectivity matrix and a rescaling of activation levels.
} 
clude information integration and causal density. Information integration, $\phi$, is defined as the effective information across the bipartition of a network that exhibits the least mutual information [33] and has been extended recently [31]. Here a balance between integration and segregation is captured as the lower bound of the potential for a system to integrate information. In contrast, while causal density [24] also has its foundations in information theory, it is developed from the notion of Granger causality [18] rather than mutual information. Granger causality is a statistical measure of causality in which $a$ causes $b$ if knowledge of the history of $a$ helps predict the future of $b$ more than knowledge of the past of $b$ alone. Causal density is calculated as the fraction of interactions among elements that are Granger casually significant. Again, like TSE complexity, it is argued that high causal density indicates the presence of globally integrated but dynamically independent elements [25].

Despite their wide application, a comprehensive understanding of the behaviour of these measures and how they relate to one another has yet to be established. Initial work in this vein considering the relationship between network structure, dynamics and complexity is reported in $[10,2,1]$.

\section{A Simple Model}

Here, we explore ensembles of spatially constrained networks each constructed over 128 nodes distributed uniformly in hypercubes of various dimensionality, varying the length scale of the interaction between the nodes. Note: in order to control for the average distance between nodes varying with the dimensionality, $d$, of the hypercube within which they are embedded, we preserve the average magnitude of spatial relationships between pairs of nodes by scaling all distances by $1 / \sqrt{d}$. We employ continuous-valued connection matrices to represent weighted connections between pairs of nodes given by $\omega_{i j}=\exp \left(-\left|\mathbf{r}_{j}-\mathbf{r}_{i}\right| / \sigma\right)$. Where, $\left|\mathbf{r}_{j}-\mathbf{r}_{i}\right|$ is the distance between nodes $i$ and $j$. Connection weights between pairs of nodes thus fall off exponentially with distance at a rate which is defined by the interaction length, $\sigma$.

Fig. 1 shows how complexity, $\mathcal{C}$, varies with the log of the interaction length, $\log _{10}(\sigma)$. The first point to note is that for low-dimensional spaces, complexity rises and falls with interaction length. ${ }^{3}$. As the dimensionality

\footnotetext{
${ }^{3}$ Since the covariance matrix of a 1-d lattice is of Gaussian Toeplitz form, this agrees
} 

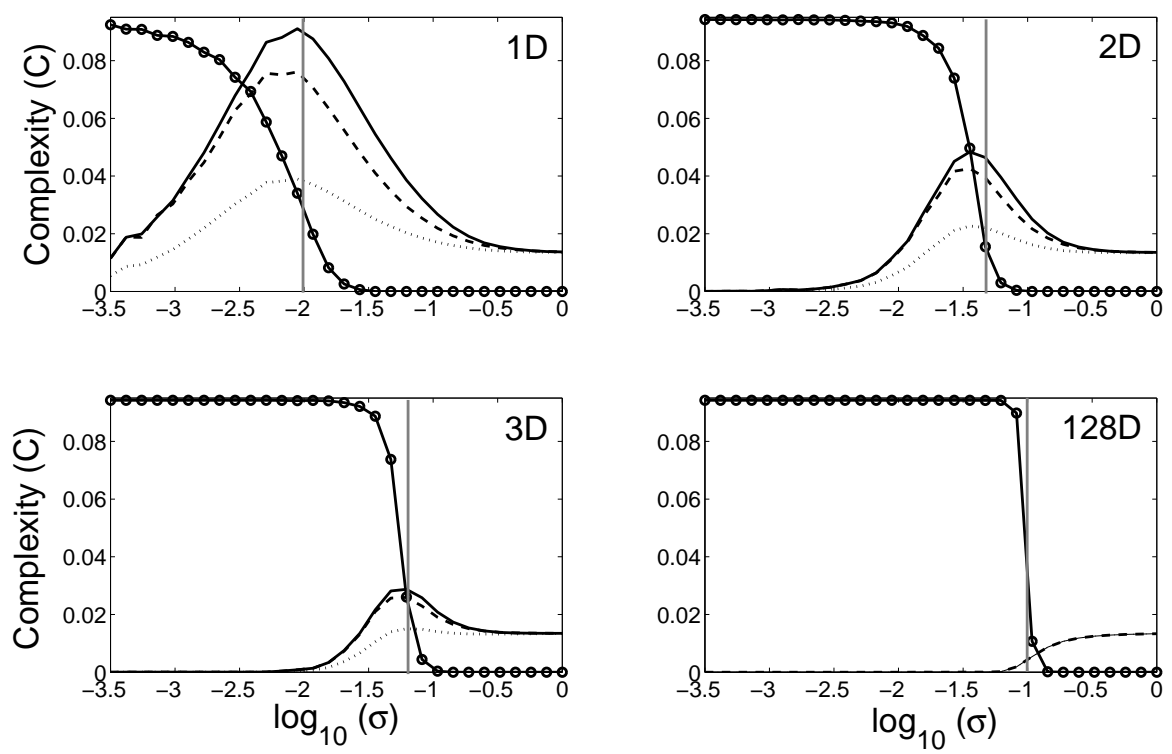

Figure 1: Plots of complexity versus the log of the interaction length, $\log _{10}(\sigma)$, for networks embedded within 1-d, 2-d, 3-d, and 128-d hypercubes. All networks comprise $N=128$ nodes, with 30 networks being generated for each data point. Solid curves represent the mean complexity, $\mathcal{C}$, of spatially embedded systems with continuous weights varying inversely with distance. Dotted and dashed lines indicates the complexity of networks derived from two null models in which aspects of spatial organisation are extinguished (see text). Grey vertical lines mark the peaks of complexity for discretised networks with the same interaction length, which agree well with the peak in complexity for the associated continuous system. The scaled number of discretised network components is also presented (circles), falling from $N$ (a totally disconnected system) to unity (a super cluster).

of the space increases, and the strength of spatial constraints weaken, peak complexity is reduced, until the contribution of space disappears.

Consider first the case of nodes embedded in a low-dimensional space. Where $\sigma$ is very small, even the closest nodes are far enough apart that the weighted connection between them is of negligible strength. Consequently the network comprises a number of effectively isolated units with low interaction complexity due to the lack of network integration. As the interaction length

with previous results demonstrating that scaling in such matrices is associated with a rise and fall in complexity [34]. 
increases, connection weights between nearby nodes begin to increase, and islands of strongly connected nodes are obtained. As a consequence, interaction complexity increases. Eventually, interaction length increases to the extent that all network nodes are close enough to each other to be strongly connected. Although the dynamics on such a network are strongly integrated at the level of the whole, interaction complexity is low since there is little or no functional segregation at the level of the parts.

Each plot in fig. 1 also presents values of complexity for two null models in which some aspect of the spatial structure inherent in the original spatially embedded networks is extinguished. In this way we are able to decompose the contribution of spatial embedding to a network's interaction complexity. First, dotted lines represent the complexity of networks in which each node retains the same distribution of afferent connection strengths as in the original network, but these weighted connections are randomly assigned rather than determined by spatial proximity. To achieve this, the entries of each row in the original weight matrix are shuffled, preserving the values of a node's afferent weights (and their sum) but assigning them at random to the population of nodes. Dashed lines represent the complexity of networks generated by a second null model in which the connection strengths of the original spatial networks are shuffled as before, but in a manner that preserves reciprocity (i.e., $\omega_{i j}=\omega_{j i}$ ). Should a shuffle swap matrix element $\omega_{i j}$ with $\omega_{i^{\prime} j}$, we must also swap elements $\omega_{j i}$ and $\omega_{j i^{\prime}}$. Note: in this case the sum of the magnitude of the afferent weights may not be preserved.

To a significant degree, the effect of spatial organisation on complexity is clearly accounted for by the reciprocal nature of spatial interactions (and to a larger degree than the mere distribution of afferent weights). However, particularly in low dimensions, the impact of spatial constraints exceeds that of mere reciprocity, suggesting that higher-order structures are significant (see [2] for a complete account).

Why do we see a peak in complexity at a particular length scale for each value of $d$ ? In fact, this peak coincides with a particular degree of network "connectance". To demonstrate this, we discretise each weighted, spatially embedded network, by reinterpreting each entry in the weight matrix as the probability that a pair of nodes will be connected with a weight equal to unity. In this way, each continuous matrix can be mapped to an ensemble of binary networks from which a random sample can be drawn and their properties calculated. For each binary network, we enumerate the number of disconnected network components (isolated fragments of network). As 
this value falls to unity with increasing $\sigma$, the graph is becoming completely connected, indicating the onset of a single giant component or super-cluster [9, 39]. Fig. 1 shows that maximal complexity coincides with the onset of this giant component in the binary ensembles. The grey vertical line also indicates that maximal complexity of the binary networks themselves agree with that of the continuous weighted networks from which they are derived. These results suggest that complexity is associated with the achievement of a single strongly coupled component in a continuous network. Furthermore the interaction length required for onset of the strong component (and thus high complexity) increases with increasing spatial dimensionality.

\section{Discussion}

Results here demonstrate that spatial constraints on connectivity contribute directly to interaction complexity. A network comprising a uniform random distribution of locally connected nodes enjoys increased complexity as a result of the strong spatial constraints imposed by a low-dimensional embedding. As these constraints are relaxed (by increasing the dimensionality of the space) or eroded (by shuffling the connection strengths), complexity falls. The pairwise reciprocity of spatial network connectivity (itself stemming from the fact that the distance from node $i$ to node $j$ must be equivalent to the distance in the reverse direction) is strongly implicated in the elevated complexity of spatially embedded networks, but does not entirely account for it. Rather, the property stems from spatial embedding imposing correlations at several topological scales ${ }^{4}$.

Interestingly, our results also suggest that high network complexity is associated with the onset of a strongly coupled super-cluster. The fact that the coupling strength required for its onset is much smaller in networks embedded within low-dimensional spaces suggests that stronger spatial constraints may make high complexity achievable with fewer/weaker network connections.

Open questions that could be addressed in further work include the following. Which graph-theoretic properties of the supercluster are associated with high complexity, e.g., it's size, clustering coefficient, modularity, etc? To what extent do the results presented here carry over to networks where,

\footnotetext{
${ }^{4}$ In [3] we are able to show, using a graph-theoretic analysis, that the TSE complexity measure is directly dependent on the frequency of loop motifs within the network.
} 
in general, $\omega_{i j} \neq \omega_{j i}$, but there remains an influence of spatial separation on node connectance.

\section{Conclusion}

Here we have demonstrated that spatially constrained network topologies exhibit complexity that differs from equivalent random non-spatial graphs. We have shown how spatial structure can impact on interaction complexity via its influence on topological structure. In summary, the inherent constraints imposed on a system by projecting it into a low-dimensional space can be enabling for complexity in that these constraints predispose systems to exhibit elevated levels of complex interactivity for free.

\section{References}

[1] Barnett, L., Barrett, A., Seth, A. K., in press. Granger causality and transfer entropy are equivalent for gaussian variables. Physical Review Letters.

[2] Barnett, L., Buckley, C. L., Bullock, S., 2009. Neural complexity and structural connectivity. Phys. Rev. E 79 (5), 051914.

[3] Barnett, L., Buckley, C. L., Bullock, S., in preparation. On a graph theoretic interpretation of neural complexity. Phys. Rev. E.

[4] Barnett, L., Di Paolo, E., Bullock, S., 2007. Spatially embedded random networks. Phys. Rev. E 76 (5), 056115.

[5] Beer, R. D., 1995. On the dynamics of small continuous-time recurrent neural networks. Adaptive Behavior 3 (4), 469-509.

[6] Beer, R. D., 2006. Parameter space structure of continuous-time recurrent neural networks. Neural Computation 18 (12), 3009-3051.

[7] Boccaletti, S., Latora, V., Moreno, Y., Chavez, M., Hwang, D.-U., 2006. Complex networks: Structure and dynamics. Phys. Rep. 424, 175-308.

[8] Boerlijst, M. C., Hogeweg, P., 1991. Spiral wave structure in pre-biotic evolution: Hypercycles stable against parasites. Physica D 48, 17-28.

[9] Bollobás, B., 1985. Random Graphs. Academic. 
[10] Buckley, C. L., Bullock, S., 2007. Spatial embedding and complexity: The small world is not enough. In: Almeida e Costa, F., Rocha, L. M., Costa, E., Harvey, I., Coutinho, A. (Eds.), Proceedings of the Ninth European Conference on Artificial Life. Springer, pp. 986-995.

[11] Bullock, S., Buckley, C. L., 2009. Embracing the "tyranny of distance": Space as an enabling constraint. Technoetic Arts 7, 141-152, special issue on Living Buildings: Plectic Systems Architecture.

[12] Buzsáki, G., Geisler, C., Henze, D. A., Wang, X.-J., 2004. Circuit complexity and axon wiring economy of cortical interneurons. Trends in Neuroscience 27 (4), 95-100.

[13] Cover, T. M., Thomas, J. A., 1991. Elements of Information Theory. Wiley-Interscience.

[14] Di Paolo, E. A., 2000. Ecological symmetry breaking can favour the evolution of altruism in an action-response game. Journal of Theoretical Biology 203, 135-152.

[15] Eigen, M., Schuster, P., 1979. The hypercycle: A principle of natural self-organization. Springer.

[16] Friston, K. J., Tononi, G., Sporns, O., Edelman, G. M., 1995. Characterising the complexity of neuronal interactions. Human Brain Mapping 3 (4), 302-314.

[17] Galán, R. F., 2008. On how network architecture determines the dominant patterns of spontaneous neural activity. PLoS ONE 3 (5), e2148.

[18] Granger, C. W. J., 1969. Investigating causal relations by econometric models and cross-spectral methods. Econometrica 37 (3), 424-438.

[19] Kauffman, S., 1993. The Origins of Order. Oxford University Press, Oxford.

[20] Langton, C. G., 1990. Computation at the edge of chaos. Physica D 42, $12-37$.

[21] Lewis, T. G., 2009. Network Science: Theory and Applications. Wiley. 
[22] Newman, M. E. J., 2003. The structure and function of complex networks. Siam Review 45 (3), 167-256.

[23] Penrose, M., 2003. Random Geometric Graphs. Oxford University Press.

[24] Seth, A. K., 2005. Causal connectivity of evolved neural networks during behavior. Network: Computation in Neural Systems 16 (1), 35-54.

[25] Seth, A. K., 2009. Explanatory correlates of consciousness: Theoretical and computational challenges. Cognitive Computation 1 (1), 50-63.

[26] Seth, A. K., Edelman, G. M., 2004. Environment and behaviour influence and complexity of evolved neural networks. Adaptive Behaviour $12(1), 5-21$.

[27] Shepherd, G. M., 2004. The Synaptic Organization of the Brain, 5th Edition. Oxford University Press, Oxford.

[28] Sporns, O., Tononi, G., Edelman, G. M., 2000. Connectivity and complexity: the relationship between neuroanatomy and brain dynamics. Neural Networks 13 (8-9), 909-922.

[29] Sporns, O., Tononi, G., Edelman, G. M., 2000. Theoretical neuroanatomy: Relating anatomical and functional connectivity in graphs and cortical connection matrices. Cerebral Cortex 10 (2), 127-141.

[30] Stam, C., 2005. Nonlinear dynamical analysis of EEG and MEG: Review of an emerging field. Clinical Neurophysiology 116 (10), 2266-2301.

[31] Tononi, G., 2008. Consciousness as integrated information: A provisional manifesto. The Biological Bulletin 215 (3), 216.

[32] Tononi, G., Edelman, G. M., 1998. Conciousness and complexity. Science 282 (5395), 1846.

[33] Tononi, G., Sporns, O., 2003. Measuring information integration. BMC Neuroscience 4 (1), 31-51.

[34] Tononi, G., Sporns, O., Edelman, G. M., 1994. A measure for brain complexity: Relating functional segregation and integration in the nervous system. Proc. Natl. Acad. Sci. 91, 5033-5037. 
[35] Tononi, G., Sporns, O., Edelman, G. M., 1996. A complexity measure for selective matching of signals by the brain. Proc. Natl. Acad. Sci. 93, $3422-3427$.

[36] Tononi, G., Sporns, O., Edelman, G. M., 1999. Measures of degeneracy and redundancy in biological networks. Proc. Natl. Acad. Sci. 96, 32573262 .

[37] Travers, J., Milgram, S., 1969. An experimental study of the small world problem. Sociometry 32 (4), 425-443.

[38] Uhlenbeck, G. E., Ornstein, L. S., 1930. On the theory of brownian motion. Phys. Rev. 36, 823-841.

[39] Watts, D. J., 1999. Small Worlds. Princeton University Press, Princeton.

[40] y Cajal, S. R., 1904/1994. Histology of the Nervous System of Man and Vertebrates. Oxford University Press, Oxford. 